J Phys Chem Lett. 2016 July 07; 7(13): 2544-2548. doi:10.1021/acs.jpclett.6b01024.

\title{
Saccharides as Perspective Immobilizers of Nucleic Acids for Room-Temperature Structural EPR Studies
}

\author{
Andrey A. Kuzhelev1,4,\#, Georgiy Yu. Shevelev $2,4, \#$, Olesya A. Krumkacheva ${ }^{3,4}$, Victor M.

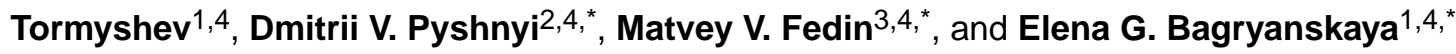 \\ ${ }^{1}$ N.N. Vorozhtsov Novosibirsk Institute of Organic Chemistry SB RAS, Novosibirsk 630090, \\ Russia \\ ${ }^{2}$ Institute of Chemical Biology and Fundamental Medicine SB RAS, Novosibirsk 630090, Russia \\ 3International Tomography Center SB RAS, Novosibirsk 630090, Russia \\ ${ }^{4}$ Novosibirsk State University, Novosibirsk 630090, Russia
}

\begin{abstract}
Pulsed Dipolar Electron Paramagnetic Resonance (EPR) spectroscopy is a powerful tool for structural studies of biomolecules and their complexes. This method, whose applicability has been recently extended to room temperatures, requires immobilization of studied biosystem to prevent averaging of dipolar couplings; at the same time, the modification of native conformations by immobilization must be avoided. Disaccharide trehalose perfectly fulfils both purposes for proteins; however its applicability for nucleic acids was questionable and not investigated so far. In this work we provide first demonstration of room-temperature EPR distance measurements on model spin-labeled DNA duplexes immobilized in trehalose, sucrose and glucose. We have analyzed different ways of sample dehydration and have discovered a reliable approach that keeps structural conformation and unity of the complex. The obtained spin-spin distances in all three saccharides agree well with our previous data using ion-exchange sorbent; however among glucose, sucrose and trehalose the latter gives the best accuracy. Finally, room-temperature electron spin dephasing time of triarylmethyl label in trehalose is noticeably longer compared to glucose, sucrose and other immobilizers used previously, thus providing a longer range of available distances. Therefore, saccharides, and especially trehalose, can be efficiently used as easy-to-handle immobilizers of nucleic acids and their complexes, mimicking native conditions and allowing broad range of structural EPR studies at room temperatures.
\end{abstract}

\section{Graphical abstract}

\footnotetext{
Corresponding Authors: egbagryanskaya@nioch.nsc.ru (E.G.B.), mfedin@tomo.nsc.ru (M.V.F.), pyshnyi @ niboch.nsc.ru (D.V.P.). \#these authors contributed equally

Supporting Information available: DQC time traces for sample dried at $303 \mathrm{~K}$. Thermal denaturation experiments. Circular Dichroism experiments. Relaxation curves. X-band DQC and DEER time traces. Comparison of DQC and sifter experiments at $298 \mathrm{~K}$. General EPR settings. This material is available free of charge via the Internet at http://pubs.acs.org.

NOTES

The authors declare no competing financial interests.
} 


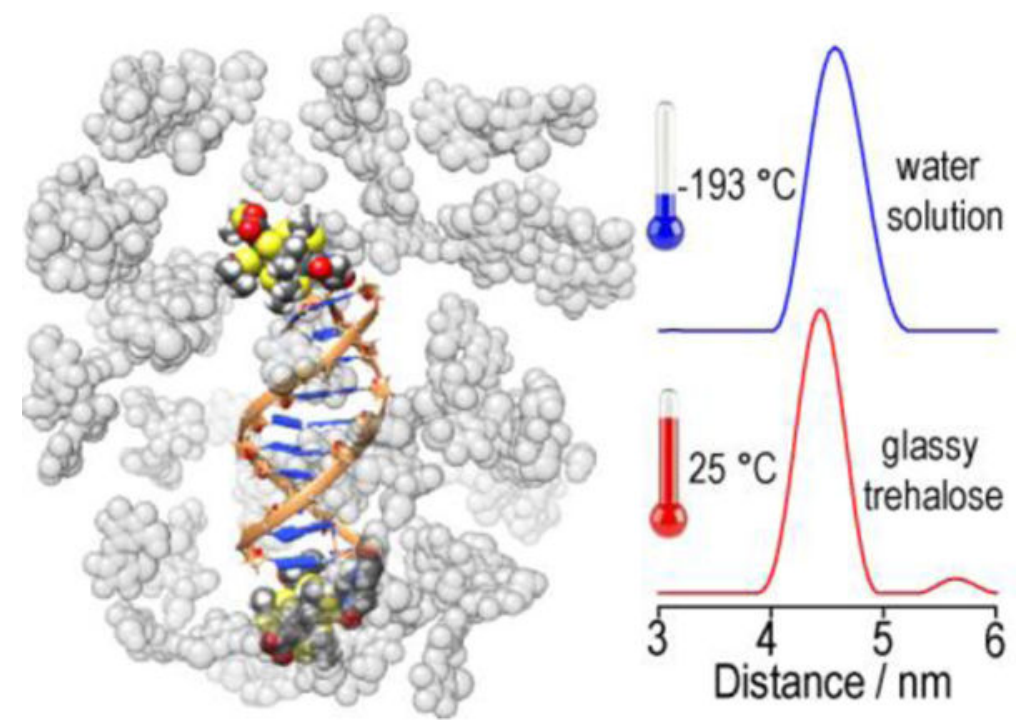

Structural studies of biomolecules and their complexes on the nanoscale are vital for understanding of many physiologically-important processes. Along with X-ray diffraction, ${ }^{1}$ cryoelectronic microscopy, ${ }^{2}$ Foerster Resonance Energy Transfer (FRET), ${ }^{3-4}$ NMR $^{1,5}$ and some other techniques, such structural information is provided by pulse dipolar Electron Paramagnetic Resonance (EPR) spectroscopy. ${ }^{6-13}$ In this approach, as a rule, two spin labels are synthetically attached to the desired positions in biosystem and dipolar coupling between them is measured, thus providing a spin-spin distance within $\sim 1.5-10 \mathrm{~nm} .{ }^{14-15}$ The range of available distances is limited by electron spin dephasing time $\left(T_{\mathrm{m}}\right)$, an internal property of spin label; the longer $T_{\mathrm{m}}$ the longer spin-spin distances can be obtained. In addition, the system under study has to be immobile on the timescale of EPR experiment to prevent averaging of dipolar interaction. The overwhelming majority of pulse dipolar EPR studies performed up to date used nitroxide spin labels, ${ }^{16-18}$ with recently emerged alternatives including $\mathrm{Gd}^{3+}, \mathrm{Mn}^{2+}, \mathrm{Cu}^{2+}$ and some other paramagnetic tags. ${ }^{19-21}$ For such spin labels long enough $T_{\mathrm{m}}$ and immobilization of studied biosystem are simultaneously achieved by shock-freezing of the sample and performing measurements at cryogenic temperatures. It is generally believed that shock-freezing captures the native conformation (or distribution of conformations) and therefore the obtained structural data closely corresponds to that in natural conditions. Despite of such expectations, which are very difficult to verify experimentally and ensure they are always met, it is highly desirable to have a versatile tool for performing similar measurements at room/physiological temperatures.

A significant breakthrough towards this goal was recently achieved by developing triarylmethyl (TAM) spin labels with microsecond-scale $T_{\mathrm{m}}$ times in water solutions at ambient temperatures. ${ }^{22-23}$ Distance measurements in water solution were first demonstrated at $4{ }^{\circ} \mathrm{C}$ for the TAM-labeled protein immobilized on a sepharose support, ${ }^{24}$ and then at $37^{\circ} \mathrm{C}$ for the TAM-labeled DNA duplex immobilized on ion-exchange sorbent. ${ }^{25}$ However, the immobilization approaches used in both of these studies were not really versatile, and development of more universal immobilization strategies applicable for 
a wide variety of biological systems and maintaining naturally occurring conformations would be highly appreciated.

One of the opportunities in this regard is provided by disaccharide trehalose, as was recently realized and demonstrated on nitroxide-labeled protein at room temperature. ${ }^{26}$ Trehalose can be found at high amounts in many anhydrobiotic organisms (fungi, certain yeast and several species of nematodes) capable of surviving severe dehydratation. ${ }^{27}$ Cryoprotective properties of trehalose originate from its ability to store considerable amounts of water molecules even after extensive dehydration and mimic the presence of water by creating the networks of hydrogen bonds. ${ }^{28-29}$ As a result, proteins in dehydrated trehalose can be stored for a long time and restore their functional properties after rehydration. ${ }^{30}$ There are many reports on the use of trehalose for successful storage of proteins, and it is now evident that spin-labeled proteins can be immobilized and studied in dehydrated trehalose at room temperatures. ${ }^{26}$ However, similar application of trehalose to nucleic acids and their complexes has not been addressed so far and is generally questionable, since corresponding complexes may fall apart or perturb their structure at low concentrations of water in dehydrated trehalose. For instance, previous study of spin-labeled DNA duplex in trehalose at room temperature was not successful due to the short phase memory time of nitroxide labels and partial denaturation of double-stranded (ds) DNA. ${ }^{31}$

In the present work, we report the first detailed investigation of trehalose as a perspective immobilizing agent of spin-labeled nucleic acids for pulse EPR distance measurements at room temperatures. To gain deeper insights into the immobilizing properties of trehalose, we also investigate two other saccharides of close structure, sucrose and glucose, and compare their performance with trehalose.

As a model system for pulse EPR distance measurements we use here the 10-mer DNA duplex doubly spin-labeled with TAM radicals (Figure 1). ${ }^{25}$ First, aqueous solution of this duplex saccharide was prepared (more details in Experimental Section). Then, two different procedures of obtaining glassy state were tested. The first approach used slow drying of the solution deposited on a glass plate at room temperature. However, following EPR studies of the obtained sample did not detect any dipolar oscillations, neither using Double Quantum Coherence $\left(\mathrm{DQC}^{11}\right)$ at room temperature nor using Double Electron-Electron Resonance (DEER or PELDOR ${ }^{13}$ ) at $50 \mathrm{~K}$ (SI Figure S1). Interestingly, when water was added to the same sample and solution was frozen again, DQC/DEER oscillations were readily detected (SI Figure S1), implying that spin-labeled single DNA strands (ssDNA) retained their original structure and rearranged again into dsDNA in water. To gain deeper understanding of these processes, we have studied the influence of saccharides on thermal stability of another model 15-bp fluorescently-labeled DNA duplex using thermal denaturation with fluorescent detection (SI Figure S2). The significant decrease of the melting temperature of the duplex was found in all saccharides, whose concentrations were varied from 0 to $1.8 \mathrm{M}$. Thus, slow evaporation of water from the saccharide solution leads to denaturation of the dissolved dsDNA. This conclusion is furthermore approved by Circular Dichroism (CD) studies of wet and deeply dried films, showing that dry samples contain single-stranded DNA (SI Figure S3 and Ref. 32). Thus, slow drying of trehalose/water solution of dsDNA results in its denaturation with a formation of ssDNA. 
Since the above method of slow drying was not suitable, we developed another approach. A trehalose/water solution of DNA duplex was first shock-frozen in liquid nitrogen, and then lyophilized in the frozen state under medium vacuum (more details in Experimental section). After such "mild" lyophilization we used additional high vacuum pumping to deoxygenate the sample and obtain longer $T_{\mathrm{m}}$ values. The room-temperature $T_{\mathrm{m}}$ noticeably increased from $1.1 \mu$ s (after "mild" step) to $2.2 \mu$ s (after pumping at $5 \cdot 10^{-8}$ bar) (SI Figure S4), whereas spin-spin distances remained unchanged upon additional pumping (as revealed afterwards by EPR).

Importantly, we have found that preventing the sample melting during the first-step lyophilization is the most critical factor for the developed procedure. For instance, if the sample was partly melted during lyophilization, we obtained spin-spin distance of $4.0 \mathrm{~nm}$, whereas the significantly larger value of $4.6 \mathrm{~nm}$ was found previously for the same duplex (i) in frozen water/glycerol solution at $80 \mathrm{~K}$, or (ii) in liquid water at $310 \mathrm{~K}$ immobilized on ion-exchange sorbent. ${ }^{25}$ Evidently, dehydration of the solid-state (frozen) sample keeps native structure of the duplex (sketched in Figure 2), whereas water evaporation from the liquid state does not, possibly due to the larger flexibility of DNA in solution. Note that although the sample freezing was not fully avoided in the developed approach, the trehalose itself is a good cryoprotector, ${ }^{33}$ diminishing the impact of freezing, and at the same time allowing the following studies at room temperature.

The room-temperature values of $T_{\mathrm{m}}$ were found different in trehalose ( $\left.2.2 \mu \mathrm{s}\right)$, sucrose (1.3 $\mu \mathrm{s})$ and glucose $(1.2 \mu \mathrm{s})$, being maximum for the trehalose sample. It is noteworthy that $T_{\mathrm{m}}$ value in dehydrated trehalose is longer than previously obtained $T_{\mathrm{m}}$ for the same duplex immobilized on ion-exchange sorbent NucleosilDMA $(1.4 \mu \mathrm{s}) .{ }^{25}$ The observed differences in $T_{\mathrm{m}}$ values may be caused by (i) different degree of immobilization in different media, or (ii) different extent of deoxygenation that can be achieved using applicable techniques (lowvacuum "freeze-pump-thaw" cycles ${ }^{25}$ vs. high-vacuum pumping), or (iii) inhomogeneous distribution of spin-labeled molecules in the volume of some immobilizers. ${ }^{34}$ To verify the first reason (i), it is instructive to compare $T_{\mathrm{m}}$ values at X- and Q-bands. Recently it was shown that room-temperature $T_{\mathrm{m}}$ values of TAMs are shorter at Q-band compared to X-band if the radical is partly mobile and thus its g-anisotropy is modulated. ${ }^{35}$ Contrary, in case of perfectly immobilized TAM label one expects a suppression of this mechanism and much weaker dependence of $T_{\mathrm{m}}$ on magnetic field. For all three saccharides we obtained the same $T_{\mathrm{m}}$ values at $80 \mathrm{~K}$ at $\mathrm{X} / \mathrm{Q}$ bands (Table 1 ). At the same time, the corresponding roomtemperature $T_{\mathrm{m}}$ values at X-band are larger than those measured at Q-band. This indicates that TAM labels, which are completely immobilized at $80 \mathrm{~K}$, still exhibit some motions/ librations at room temperature, and thus the use of X-band frequency is superior for roomtemperature pulse dipolar EPR (PDS). ${ }^{35}$

Single narrow EPR line of TAM label favors the applications of single-frequency PDS methods, such as DQC ${ }^{11}$ or SIFTER. ${ }^{10}$ Figure 2 shows distance distributions obtained for DNA duplex immobilized in trehalose, sucrose and glucose, whereas Table 2 reports the corresponding mean distances $\langle\mathrm{r}\rangle$ and standard deviations $\sigma$. For comparison, Figure 2 also presents previously obtained data $(<\mathrm{r}>=4.61 \mathrm{~nm}, \sigma=0.21 \mathrm{~nm})$ for the same duplex immobilized using NucleosilDMA in water. ${ }^{25}$ Note that shorter $T_{\mathrm{m}}$ values and difficulties 
with sample preparation/transfer in case of sucrose and glucose did not allow roomtemperature distance measurements of satisfactory quality (see Experimental Section); however, valid comparison of different saccharides still can be made at $80 \mathrm{~K}$.

As an additional confirmation of successful room-temperature DQC measurement in trehalose, we performed DEER measurements on the same sample at $80 \mathrm{~K}$ (Figure 2). Distance distributions obtained using DQC at $298 \mathrm{~K}$ and DEER at $80 \mathrm{~K}$ are very similar, and distribution widths $\sigma$ are very close. The latter implies that existing motion of TAM-labels at room temperature has a negligible influence on the obtained distance distribution. In addition, room-temperature $\sigma$ in trehalose $(0.24 \mathrm{~nm})$ is nearly the same as that in water $(0.21$ $\mathrm{nm}$ ) measured earlier using NucleosilDMA as immobilizer. ${ }^{25}$ This indicates that TAM-labels retain the ability to occupy "capping" positions of the terminal base pairs of the duplex. ${ }^{36}$ Furthermore, previously reported mean distance varies between 4.52 and $4.61 \mathrm{~nm}$ for different PDS techniques, ${ }^{25,36}$ which allows neglecting the difference between $\langle\mathrm{r}\rangle$ values obtained here in trehalose $(4.43-4.47 \mathrm{~nm})$ and previously in water.

In order to compare suitability of trehalose, sucrose and glucose as immobilizers of dsDNA, we used X-band DQC at $80 \mathrm{~K}$ (Figure 3). Standard deviations in sucrose $(\sigma=0.27 \mathrm{~nm})$ and glucose $(\sigma=0.28 \mathrm{~nm})$ are larger compared to trehalose $(\sigma=0.22 \mathrm{~nm})$. In fact, the broadening of the distance distribution in sucrose/glucose owes to the onset of short-distance shoulder, whereas the long-distance edge of the distribution remains practically the same as that in trehalose. The enhancement of contribution from short distances implies that some fraction of the duplexes fall apart, or get perturbed or frayed. Therefore, among three studied saccharides trehalose shows the best performance as a non-perturbing DNA immobilizer for following room-temperature PDS measurements.

Note that another single-frequency PDS method SIFTER ${ }^{10}$ can also be used for roomtemperature distance measurements on doubly TAM-labeled DNA (SI Figure S6). Remarkably, SIFTER allows improving the signal-to-noise ratio (SNR) by roughly a factor of two compared to DQC. Such trend agrees well with recently published four-fold gain using SIFTER vs. DQC achieved at Q-band at $50 \mathrm{~K} .{ }^{38}$ Thus, even better accuracy of roomtemperatures distance measurements on doubly TAM-labeled biomolecules can be reached using SIFTER.

In conclusion, herewith we have demonstrated the effective approach for immobilization of nucleic acids in trehalose, which allows pulse dipolar EPR studies of unperturbed (native) structures at room temperatures. We have shown that the room-temperature distance distribution in doubly TAM-labeled dsDNA in trehalose is close to that measured previously using ion-exchange sorbent in water. Compared to two other saccharides glucose and sucrose, glassy trehalose provides the best agreement with distances found in water. In addition, sample preparation using trehalose is much easier-to-handle compared to glucose and sucrose. Finally, electron spin dephasing time of TAM-labeled dsDNA in trehalose is $\sim 1.5$ times larger than that in all other immobilizers studied previously. Consequently, implementation of TAM radicals as spin labels and glassy trehalose as immobilizing medium provides a new promising strategy for studying native structures of nucleic acids and their protein complexes at room temperature using EPR. 


\section{EXPERIMENTAL SECTION}

\section{Sample preparation}

Synthesis of TAM-labeled DNA duplexes was made according to previously developed procedure. ${ }^{25} \mathrm{D}(+)$ Trehalose dihydrate, $\mathrm{D}(+)$ Glucose, $\mathrm{D}(+)$ Saccharose (all Sigma-Aldrich, USA, purity $299 \%$ ) were used as immobilizers of spin-labeled DNA duplexes. The aqueous solution of TAM-labeled DNA $\left(\mathrm{C}_{\text {total }}=2.4 \times 10^{-5} \mathrm{M}\right)$ in $10 \mathrm{mM}$ sodium cacodilate $(\mathrm{pH} 7.0)$, $0.65 \mathrm{M}$ saccharide was prepared for following dehydration. In the first drying approach $60 \mu \mathrm{l}$ drop of solution has been deposited on a quartz plate and dried in a dessicator with $\mathrm{P}_{2} \mathrm{O}_{5}$ for about 3 days (the same approach was used for $\mathrm{CD}$ experiments). The second approach consisted of two steps. The first one used the shock-freezing of the prepared solution in $2 \mathrm{ml}$ Eppendorf tube, then quick transfer to the dessicator and following lyophilization under vacuum of $10^{-3}$ bar for two hours. At the second step the sample was transferred into quartz EPR tube (OD $3.8 \mathrm{~mm}$, ID $2.8 \mathrm{~mm}$ for X-band; OD $2.8 \mathrm{~mm}$, ID $1.8 \mathrm{~mm}$ for Q-band) and dried using turbomolecular pump $\left(\sim 5 \cdot 10^{-8}\right.$ bar $)$ for 24 hours to remove remaining oxygen and enhance relaxation times. In case of trehalose the first step yielded fine powder that was easily transferred into the EPR tube. However, in case of sucrose and glucose the first step yielded extremely viscous and sticky solutions, which were very difficult to transfer to the EPR tubes in sufficient amount.

\section{EPR Measurements}

All experiments were performed using X/Q-band (9/34 GHz) Bruker Elexsys E580 spectrometer equipped with Oxford flow helium cryostat and temperature control system. Electron spin dephasing times $\left(T_{\mathrm{m}}\right)$ were measured at the maximum of the spectrum using a standard two-pulse electron spin echo (ESE) sequence with $\pi / 2$ and $\pi$ pulse lengths being 10/20 ns (X-band) and 100/200 ns (Q-band). The obtained room-temperature echo decays were approximated by monoexponential function. DEER measurements used a standard four-pulse DEER sequence ${ }^{39}$ with pulse lengths 60/120 ns for probe and $120 \mathrm{~ns}$ for pump frequency. We used the ${ }^{13} \mathrm{C}$ satellite for observing and the main line of ${ }^{12} \mathrm{C}$ for pumping. DQC measurements were done at the maximum of the spectrum and used original six-pulse sequence ${ }^{11-12}$ with pulse lengths $10 / 20 \mathrm{~ns}$ (for $\pi / 2$ and $\pi$ pulses) and 64-step phase cycling. The obtained DEER and DQC time traces were analyzed using DeerAnalysis program. ${ }^{37}$ More details are given in Supporting Information.

\section{Supplementary Material}

Refer to Web version on PubMed Central for supplementary material.

\section{Acknowledgments}

This work was supported by Russian Science Foundation (no. 14-14-00922).

\section{References}

1. Torres J, Stevens TJ, Samso M. Membrane proteins: the 'Wild West' of structural biology. Trends Biochem Sci. 2003; 28:137-144. [PubMed: 12633993] 
2. Williams KA. Three-Dimensional Structure of the Ion-coupled Transport Protein NhaA. Nature. 2000; 403:112-115. [PubMed: 10638764]

3. Heuck AP, Johnson AE. Pore-forming Protein Structure Analysis in Membranes using Multiple Independent Fluorescence Techniques. Cell Biochem Biophys. 2002; 36:89-101. [PubMed: 11939373]

4. Chattopadhyay A, Raghuraman H. Application of Fluorescence Spectroscopy to Membrane Protein Structure and Dynamics. Curr Sci. 2004; 87:175-180.

5. Fernandez C, Wuthrich K. NMR Solution Structure Determination of Membrane Proteins Reconstituted in Detergent Micelles. FEBS Lett. 2003; 555:144-150. [PubMed: 14630335]

6. Jeschke G. DEER Distance Measurements on Proteins. Annu Rev Phys Chem. 2012; 63:419-446. [PubMed: 22404592]

7. Duss O, Michel E, Yulikov M, Schubert M, Jeschke G, Allain FH-T. Structural Basis of the nonCoding RNA RsmZ Acting as a Protein Sponge. Nature. 2014; 509:588-592. [PubMed: 24828038]

8. Schiemann O, Prisner TF. Long-range distance determinations in biomacromolecules by EPR spectroscopy. Q Rev Biophys. 2007; 40:1-53. [PubMed: 17565764]

9. Tsvetkov YD, Grishin YA. Techniques for EPR spectroscopy of pulsed electron double resonance (PELDOR): A review. Instrum Exp Tech. 2009; 52:615-636.

10. Jeschke G, Pannier M, Godt A, Spiess HW. Dipolar spectroscopy and spin alignment in electron paramagnetic resonance. Chem Phys Lett. 2000; 331:243-252.

11. Borbat PP, Freed JH. Multiple-quantum ESR and distance measurements. Chem Phys Lett. 1999; 313:145-154.

12. Saxena S, Freed JH. Theory of Double Quantum two-Dimensional Electron Spin Resonance with Application to Distance Measurements. J Chem Phys. 1997; 107:1317-1340.

13. Milov AD, Salikhov KM, Shirov MD. Application of PELDOR in electron-spin echo for paramagnetic center space distribution in solids. Fiz Tverd Tela. 1981; 23:975-982.

14. Borbat PP, Davis JH, Butcher SE, Freed JH. Measurement of large distances in biomolecules using double-quantum filtered refocused electron spin-echoes. J Am Chem Soc. 2004; 126:7746-7747. [PubMed: 15212500]

15. Jeschke G, Polyhach Y. Distance measurements on spin-labelled biomacromolecules by pulsed electron paramagnetic resonance. Phys Chem Chem Phys. 2007; 9:1895-1910. [PubMed: 17431518]

16. Bagryanskaya EG, Krumkacheva OA, Fedin MV, Marque SRA. Development and Application of Spin Traps, Spin Probes, and Spin Labels. Methods Enzymol. 2015; 563:365-96. [PubMed: 26478492]

17. Borbat, PP., Freed, JH. Pulse Dipolar Electron Spin Resonance: Distance Measurements. SpringerVerlag; Berlin: 2014.

18. Shelke SA, Sigurdsson ST. Site-Directed Spin Labelling of Nucleic Acids. Eur J Org Chem. 2012; 2012:2291-2301.

19. Lueders P, Jeschke G, Yulikov M. Double Electron-Electron Resonance Measured Between Gd3+ Ions and Nitroxide Radicals. J Phys Chem Lett. 2011; 2:604-609.

20. Banerjee D, Yagi H, Huber T, Otting G, Goldfarb D. Nanometer-Range Distance Measurement in a Protein Using Mn2+ Tags. J Phys Chem Lett. 2012; 3:157-160.

21. Cunningham TF, Shannon MD, Putterman MR, Arachchige RJ, Sengupta I, Gao M, Jaroniec CP, Saxena S. Cysteine-Specific Cu2+ Chelating Tags Used as Paramagnetic Probes in Double Electron Electron Resonance. J Phys Chem B. 2015; 119:2839-2843. [PubMed: 25608028]

22. Owenius R, Eaton GR, Eaton SS. Frequency (250 MHz to $9.2 \mathrm{GHz}$ ) and Viscosity Dependence of Electron Spin Relaxation of Triarylmethyl Radicals at Room Temperature. J Magn Reson. 2005; 172:168-175. [PubMed: 15589420]

23. Yong L, Harbridge J, Quine RW, Rinard GA, Eaton SS, Eaton GR, Mailer C, Barth E, Halpern HJ. Electron Spin Relaxation of Triarylmethyl Radicals in Fluid Solution. J Magn Reson. 2001; 152:156-161. [PubMed: 11531374] 
24. Yang ZY, Liu YP, Borbat P, Zweier JL, Freed JH, Hubbell WL. Pulsed ESR Dipolar Spectroscopy for Distance Measurements in Immobilized Spin Labeled Proteins in Liquid Solution. J Am Chem Soc. 2012; 134:9950-9952. [PubMed: 22676043]

25. Shevelev GY, Krumkacheva OA, Lomzov AA, Kuzhelev AA, Rogozhnikova OY, Trukhin DV, Troitskaya TI, Tormyshev VM, Fedin MV, Pyshnyi DV, et al. Physiological-Temperature Distance Measurement in Nucleic Acid using Triarylmethyl-Based Spin Labels and Pulsed Dipolar EPR Spectroscopy. J Am Chem Soc. 2014; 136:9874-9877. [PubMed: 24963806]

26. Meyer V, Swanson MA, Clouston LJ, Boratyński PJ, Stein RA, Mchaourab HS, Rajca A, Eaton SS, Eaton GR. Room-Temperature Distance Measurements of Immobilized Spin-Labeled Protein by DEER/PELDOR. Biophys J. 2015; 108:1213-1219. [PubMed: 25762332]

27. Crowe LM. Lessons from nature: the role of sugars in anhydrobiosis. Comp Biochem Physiol AMol Integr Physiol. 2002; 131:505-513. [PubMed: 11867276]

28. Allison SD, Chang B, Randolph TW, Carpenter JF. Hydrogen Bonding between Sugar and Protein is Responsible for Inhibition of Dehydration-induced Protein Unfolding. Arch Biochem Biophys. 1999; 365:289-298. [PubMed: 10328824]

29. Crowe JH, Crowe LM, Chapman D. Preservation of Membranes in Anhydrobiotic Organisms - The Role of Trehalose. Science. 1984; 223:701-703. [PubMed: 17841031]

30. Crowe LM, Crowe JH, Rudolph A, Womersley C, Appel L. Preservation of Freeze-Dried Liposomes by Trehalose. Arch Biochem Biophys. 1985; 242:240-247. [PubMed: 4051504]

31. Kuzhelev AA, Strizhakov RK, Krumkacheva OA, Polienko YF, Morozov DA, Shevelev GY, Pyshnyi DV, Kirilyuk IA, Fedin MV, Bagryanskaya EG. Room-Temperature Electron Spin Relaxation of Nitroxides Immobilized in Trehalose: Effect of Substituents Adjacent to NO-group. J Magn Reson. 2016; 266:1-7. [PubMed: 26987109]

32. Ivanov VI, Minchenkova LE, Schyolkina AK, Poletayev AI. Different Conformations of DoubleStranded Nucleic Acid in Solution as Revealed by Circular Dichroism. Biopolymers. 1973; 12:89110 .

33. Branca C, Magazu S, Maisano G, Migliardo P. Anomalous cryoprotective effectiveness of trehalose: Raman scattering evidences. J Chem Phys. 1999; 111:281-287.

34. Malferrari M, Nalepa A, Venturoli G, Francia F, Lubitz W, Mobius K, Savitsky A. Structural and dynamical characteristics of trehalose and sucrose matrices at different hydration levels as probed by FTIR and high-field EPR. Phys Chem Chem Phys. 2014; 16:9831-9848. [PubMed: 24358471]

35. Kuzhelev AA, Trukhin DV, Krumkacheva OA, Strizhakov RK, Rogozhnikova OY, Troitskaya TI, Fedin MV, Tormyshev VM, Bagryanskaya EG. Room-Temperature Electron Spin Relaxation of Triarylmethyl Radicals at the X- and Q-Bands. J Phys Chem B. 2015; 119:13630-13640. [PubMed: 26001103]

36. Shevelev GY, Krumkacheva OA, Lomzov AA, Kuzhelev AA, Trukhin DV, Rogozhnikova OY, Tormyshev VM, Pyshnyi DV, Fedin MV, Bagryanskaya EG. Triarylmethyl Labels: Toward Improving the Accuracy of EPR Nanoscale Distance Measurements in DNAs. J Phys Chem B. 2015; 119:13641-13648. [PubMed: 26011022]

37. Jeschke G, Chechik V, Ionita P, Godt A, Zimmermann H, Banham J, Timmel CR, Hilger D, Jung H. DeerAnalysis2006 - a Comprehensive Software Package for Analyzing Pulsed ELDOR Data. Appl Magn Reson. 2006; 30:473-498.

38. Akhmetzyanov D, Schöps P, Marko A, Kunjir N, Sigurdsson ST, Prisner T. Pulsed EPR DipolarSpectroscopy at Q-and G-Band on a Trityl Biradical. Phys Chem Chem Phys. 2015; 17:24446-24451. [PubMed: 26339694]

39. Pannier M, Veit S, Godt A, Jeschke G, Spiess HW. Dead-time Free Measurement of Dipole-Dipole Interactions between Electron Spins. J Magn Reson. 2000; 142:331-340. [PubMed: 10648151] 

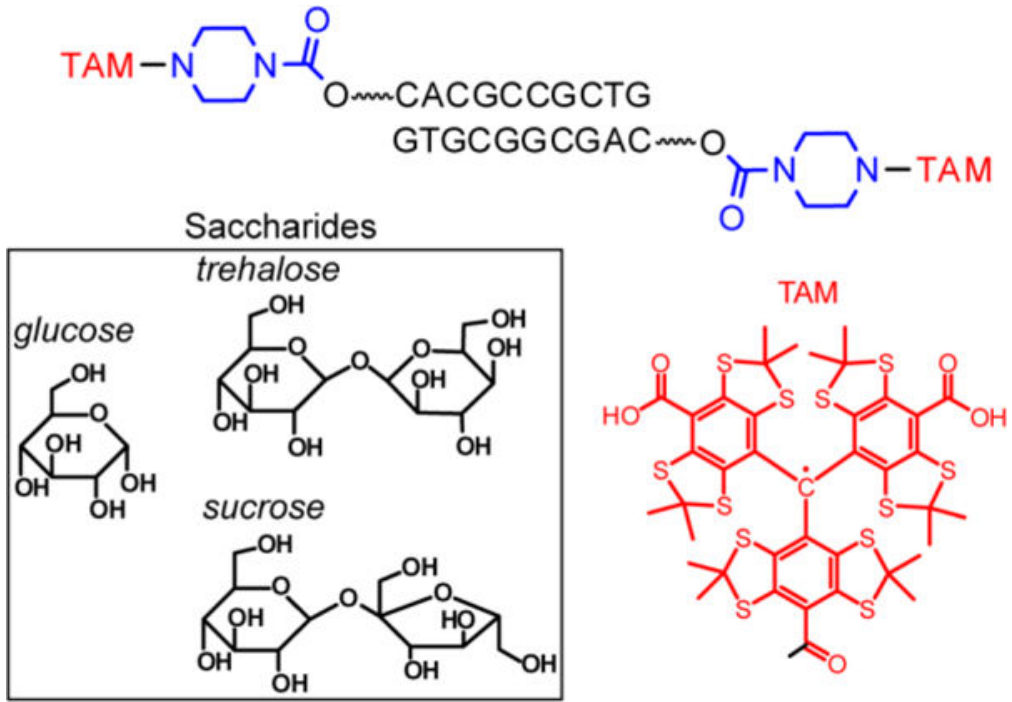

Figure 1.

Structures of studied TAM-labeled DNA duplex (top), TAM radical and saccharides used as immobilizers 


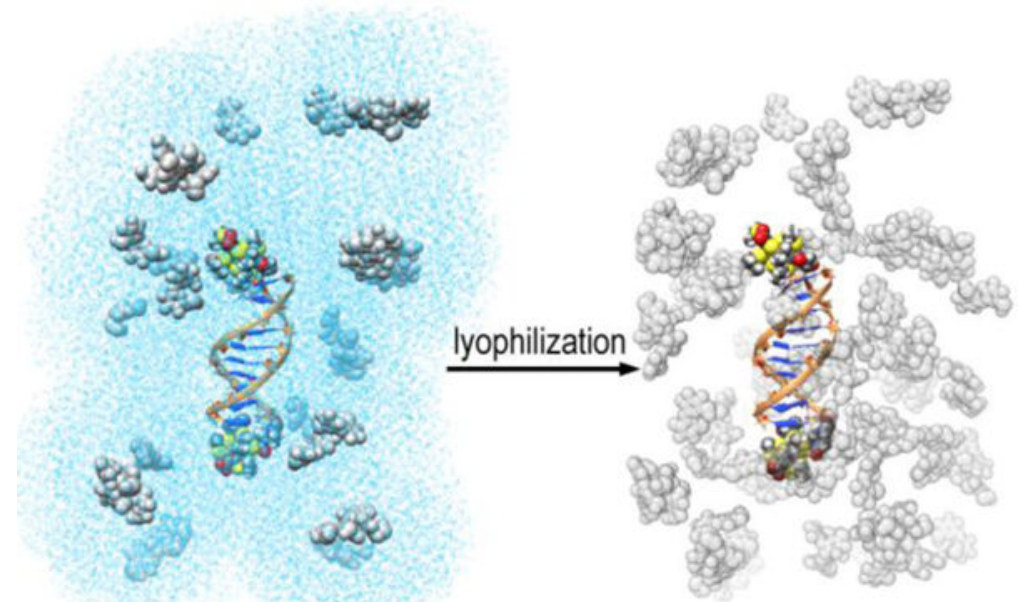

Figure 2.

Schematic representation of TAM-labeled DNA in trehalose before (left) and after (right) lyophilization. 

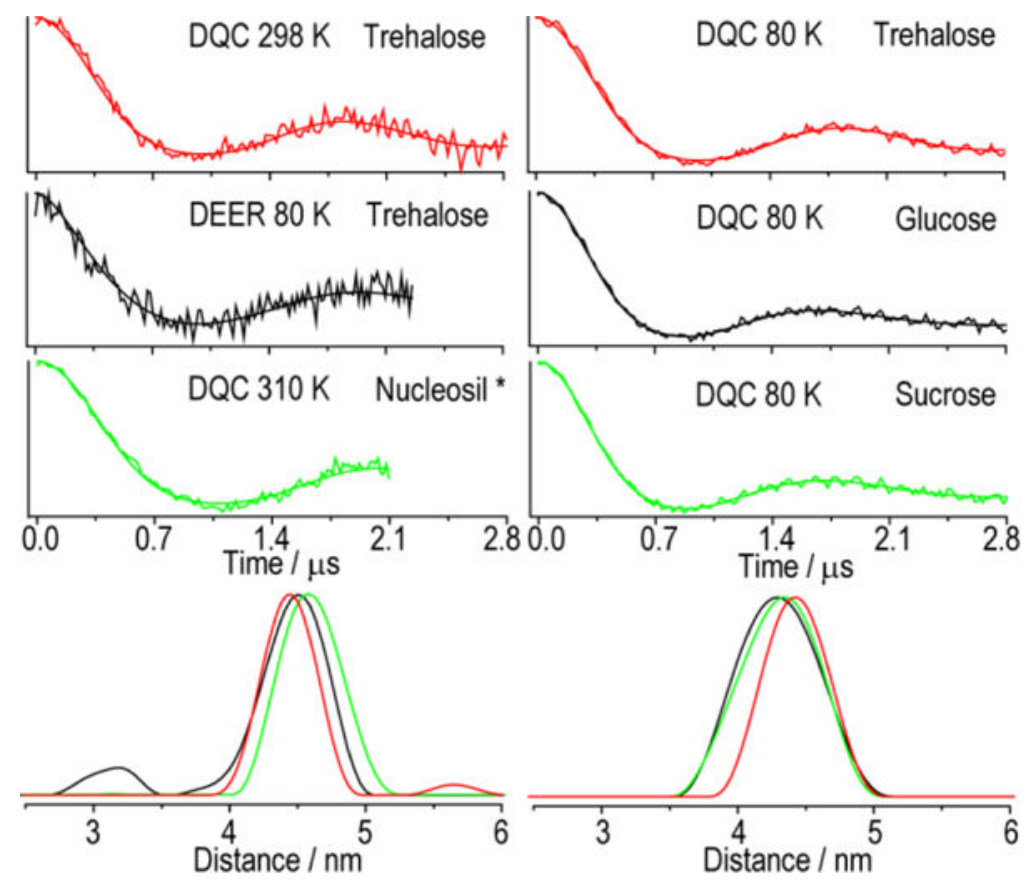

Figure 3.

(top) Background-corrected X-band DQC and DEER time traces obtained for the studied DNA duplex. The intensities are normalized (more details in SI Figure S5), temperature values, methods used and immobilizing agents are indicated. (bottom) Distance distributions obtained using DeerAnalysis. ${ }^{37}$ Tikhonov regularization parameter was 100 . (*) Background-corrected DQC time trace and corresponding distance distribution were taken from Ref. 25. 


\section{Table 1}

The phase memory times of spin labeled DNA duplexes in trehalose, sucrose and glucose at X- and Q-bands. $T_{\mathrm{m}}$ value is shown in $\mu \mathrm{s}$, corresponding temperature is indicated. Microwave frequency bands are shown in parentheses.

\begin{tabular}{ccccc}
\hline \multirow{2}{*}{ Saccharides } & \multicolumn{2}{c}{$298 \mathbf{K}$} & \multicolumn{2}{c}{$\mathbf{8 0 ~ K}$} \\
\cline { 2 - 5 } & $\boldsymbol{T}_{\mathbf{m}}(\mathrm{X})$ & $\boldsymbol{T}_{\mathbf{m}}(\mathbf{Q})$ & $\boldsymbol{T}_{\mathbf{m}}(\mathrm{X})$ & $\boldsymbol{T}_{\mathbf{m}}(\mathbf{Q})$ \\
\hline Trehalose & 2.2 & 1.2 & 3.1 & 3.1 \\
Sucrose & 1.3 & 0.8 & 2.9 & 2.9 \\
Glucose & 1.2 & 0.8 & 3.0 & 3.0 \\
\hline
\end{tabular}




\section{Table 2}

Mean Distances $\langle\mathrm{r}\rangle$ and standard deviations ( $\sigma$ ) obtained by pulse EPR for studied DNA-duplex in saccharides.

\begin{tabular}{cccc}
\hline Saccharide & \multicolumn{3}{c}{$\langle\mathbf{r}\rangle \pm \boldsymbol{\sigma}(\mathbf{n m})$} \\
\hline Trehalose & $4.43 \pm 0.24$ & DQC & $298 \mathrm{~K}$ \\
& $4.43 \pm 0.22$ & DQC & $80 \mathrm{~K}$ \\
& $4.47 \pm 0.23$ & DEER & $80 \mathrm{~K}$ \\
\hline Sucrose & $4.31 \pm 0.27$ & DQC & $80 \mathrm{~K}$ \\
\hline Glucose & $4.30 \pm 0.28$ & DQC & $80 \mathrm{~K}$ \\
\hline
\end{tabular}

